\section{Summary of: The oral health status of pre-treatment head and neck cancer patients}

\author{
S. B. Critchlow, ${ }^{* 1}$ C. Morgan ${ }^{2}$ and T. Leung ${ }^{2}$
}

\section{FULL PAPER DETAILS}

Specialist Registrar in Restorative Dentistry, ${ }^{2}$ Consultant in Restorative Dentistry, Department of Restorative Dentistry, Royal London Hospital Dental Institute, New Road, Whitechapel, London, E1 1BB

*Correspondence to Simon Bryan Critchlow Email:simon.critchlow@bartshealth.nhs.uk Refereed Paper Accepted 25 September 2013 DOI: 10.1038/sj.bdj.2013.1246 -British Dental Journal 2014; 216: E1

Background It is sometimes stated anecdotally that patients with head and neck cancer have poor oral health at the time of oncology diagnosis; however, there is little data in the literature to confirm or refute this claim. Aim To assess, in the first UK study of its type, the oral health status of 100 patients attending for a dental assessment at a single centre before head and neck cancer treatment was commenced. Subjects Sixty-six male patients and 34 female patients were included. Results Of all 100 patients, 2\% (2/100) were edentulous. Of the dentate patients, 71\% (70/98) were clinically diagnosed with periodontal disease. Fifty-one percent (50/98) had a maximum probing pocket depth in excess of $6 \mathrm{~mm}$. The mean number of carious teeth per dentate subject was 2.4 (95\% C.I. 1.6-3.1). Sixty-one percent (60/98) of dentate patients presented with one or more carious teeth. Conclusions Within this cohort, head and neck cancer patients had poor oral health at the time of oncology diagnosis, with caries and periodontal disease being important clinical issues.

The implications for the dental management of these patients are discussed.

\section{EDITOR'S SUMMARY}

There probably was a time when the differentiation of patient groups was far from clear. The majority of patients had widespread caries and/or periodontal disease and their care was consequently more run of the mill, perhaps less subtle and certainly less defined. Not so today. Various groups, types or cohorts of patients now require very specific types of care and consideration which may be due to disease patterns, overall health such as those with special care needs or socially defined groups such as prison inmates.

This research focussed on the oral health pre-treatment needs of head and neck cancer patients and found that as with other groups suffering from major life disadvantage, by dint of their conditions, their oral health was poor and to some extent neglected. No particular judgment is involved here; a range of circumstances dictates such status due to lack of knowledge, poor access, overwhelming social and medical considerations which leave the mouth as a lesser priority in the overall scheme of things and other perhaps less tangible influences.

What emerges from this research, which is a starting point for further investigation by these and hopefully other researchers too, is a pattern which dictates that we spend more time and more care in devising appropriate treatment plans for patients finding themselves in this unfortunate situation. In particular it strikes me that continuing prevention is of the essence. Root caries is mentioned in particular but the application of our full arsenal of knowledge and preventive skill is required in order to make life not just bearable and functional but to enhance it to allow as normal a routine as possible. As is also highlighted, with more patients living for longer thanks to the advances in oncology therapy more of us are likely to see them for regular care compared to the past when such treatment as they may have received would have been likely confined to more specialised units.
This provides a very valuable base from which to further explore, interrogate and improve oral care for this vulnerable group of patients.

The full paper can be accessed from the $B D J$ website (www.bdj.co.uk), under 'Research' in the table of contents for Volume 216 issue 1.

Stephen Hancocks Editor-in-Chief DOI: 10.1038/sj.bdj.2013.1244 


\section{TO ACCESS THE BDJ WEBSITE TO READ THE FULL PAPER}

- BDA Members should go to www.bda.org.

- Click the 'login' button on the right-hand side and enter your BDA login details.

- Once you have logged in click the 'BDJ' tab to transfer to the BDJ website with full access.

IF YOUR LOGIN DETAILS DO NOT WORK:

- Get a password reminder: go to www.bda.org, click the login button on the right-hand side and then click the forgotten password link.

- Use a recommended browser: we recommend Microsoft Internet Explorer or Mozilla Firefox.

- Ensure that the security settings on your browser are set to recommended levels.

IF YOU HAVE NOT YET SIGNED UP TO USE THE BDA WEBSITE:

- Go to www.bda.org/getstarted for information on how to start using the BDA website.

\section{COMMENTARY}

Superficially this appears a straight forward paper recording the dental status of patients with oral cancer but buried within it is a very important message with wide implications for primary and secondary care dental services.

Treatment paradigms have changed with many more patients receiving radiation and/or chemotherapy, (90\% of the present cohort of patients) in combination with surgery as part of their modern oral cancer treatment. The effects of chemo-radiation are more intense and leave a more vulnerable oral tissue environment with a much reduced propensity to heal. Osteoradionecrosis is now an ever present threat.

The problems presented by osteoradionecrosis are fearsome. Early lesions may respond to a drug combination of pentoxyphyline and vitamin $\mathrm{E}$ but established cases never heal. Surgical resection is all that is available with its attendant complications, human misery, prolonged hospital stay and hidden costs. The rationalisation of oral cancer care into a smaller number of specialist cancer centres has meant that oral health is no longer being overlooked. Quite the contrary, it is now likely to form part of the 'outcome assessment' by which cancer centres will be compared (head and neck surgeons' results were forcibly published this year). The object is to drive up standards by publishing comparable results.

As a cancer surgeon, I cannot emphasise enough how important the long-term dental care is to the 'cancer cured' patients. The problem comes in two forms. The hospital restorative oncology service has to address the immediate dental issues; they individualise care, decide which teeth to remove, or whether to aim for a shortened dental arch given the prospect of trismus that may limit future treatment and finally assessing likely patient compliance. The second phase comes after the patient's dental status has been optimised after treatment and at about 12-18 months responsibility of care shifts back to the primary care sector (normally a black hole).

This article charts some of the problems that have to be faced. The general condition of the patients' mouths is poor, with $70 \%$ showing evidence of periodontal disease and 50\% quite severe gum pocketing. In addition $60 \%$ of individuals have carious lesions at presentation and over 37\% have complex restorations in the form of different crown or bridges. It is widely acknowledged that the new dental epidemic in our increasingly elderly population is caries, often, but not exclusively root caries. This cohort of oral cancer patients is especially susceptible to this problem.

The authors should be congratulated on this timely publication, which lifts the lid off a very significant problem. As oncologists we can cure about 60-70\% of our patients with oral cancer but the consequential reality is that a pool of patients are gradually accumulating in the community that are vulnerable to very significant dental problems. Unfortunately, the specter of delayed healing and osteoradionecrosis in this group is an ever-present reality.

This article is a clarion call for a national debate to help quantify these problems and then establish a set of accepted standards for the effective management of this patient cohort in the primary and secondary care sectors.

\section{Professor Mark McGurk}

Department of Oral and Maxillofacial

Surgery, Guy's Hospital, London, SE1 9RT
IN BRIEF

- Reports the first UK data published on this subject.

- Highlights the poor oral health of head and neck cancer patients at the time of diagnosis and the potential impact on long-term oral health.

- Emphasises the need for comprehensive preventive regimes in this group of patients.

\section{AUTHOR QUESTIONS AND ANSWERS}

1. Why did you undertake this research?

Poor oral health is often seen in patients with head and neck cancer. Oncology surgery can leave patients with altered anatomy that can impact on oral function. In addition, many head and neck cancer patients undergo radiotherapy as part of their cancer treatment, one of the side effects of which is xerostomia. This can lead to rapid breakdown of their dentition due to caries in the post-treatment period. Since dental diseases are largely preventable, information on the pre-treatment status of this group of patients is important. A literature search yielded no data on UK head and neck cancer patients. We undertook this study in order to report some initial findings and to hopefully stimulate others to report data on this topic.

\section{What would you like to do next in this} area to follow on from this work?

It would be of interest to follow-up this cohort into the post-treatment period to see what amount of new disease, particularly caries, occurred following radiotherapy. This could then be correlated with the levels of pre-treatment disease. Another avenue would be to compare a cohort such as ours with a matched cohort of patients with no cancer diagnosis. It would also be useful to see if the results were similar on a geographically different cohort to account for regional variation. The picture is far from complete in terms of how pre-treatment oral health status affects the prognosis of the dentition in the posttreatment period. 